\title{
Ciliated adenocarcinomas of the lung: a tumor of non-terminal respiratory unit origin
}

\author{
Won Young Park ${ }^{1,4}$, Mi Hyun Kim²,4, Dong Hoon Shin ${ }^{1,3}$, Jung Hee Lee ${ }^{1}$, Kyung Un Choi ${ }^{1}$, \\ Jee Yeon Kim ${ }^{1}$, Do Youn Park ${ }^{1}$, Chang Hun Lee ${ }^{1}$ and Mee Young Sol ${ }^{1}$ \\ ${ }^{1}$ Department of Pathology, School of Medicine, Pusan National University, Yangsan, Korea; ${ }^{2}$ Department of \\ Internal Medicine, Pusan National University, Yangsan, Korea and ${ }^{3}$ Medical Research Institute, Pusan \\ National University, Yangsan, Korea
}

\begin{abstract}
Whereas most carcinomas occur through a sequential step, atypical adenomatous hyperplasia and bronchioloalveolar carcinoma pathway is known for pulmonary adenocarcinoma. This type is known as terminal respiratory unit adenocarcinoma. Based on our observation of transitions from normal ciliated columnar cells to adenocarcinoma via dysplastic mucous columnar cells, we reviewed our archive of pulmonary adenocarcinoma. Terminal respiratory unit type adenocarcinoma was defined as adenocarcinoma with type II pneumocyte, Clara cell, or bronchiolar cell morphology according to previous reports. Among 157 cases, 121 cases have been identified as terminal respiratory unit type adenocarcinoma and 36 cases as non-terminal respiratory unit type adenocarcinoma. Among non-terminal respiratory unit type adenocarcinoma, 24 cases revealed mucous columnar cell changes that were continuous with bronchial ciliated columnar cells. The mucous columnar cells became dysplastic showing loss of cilia, disorientation, and enlarged nuclei. Adenocarcinoma arose from these dysplastic mucous columnar cells and, characteristically, this type of adenocarcinoma showed acute inflammation, and honeycombing changes in the background. TTF1 immunostaining was consistently negative. In a case study with 14 males and 10 females, including 12 smokers or ex-smokers, EGFR and KRAS mutations were detected in 3 and 6 patients, respectively. We think that this kind of adenocarcinoma arising through mucous columnar cell change belongs to non-terminal respiratory unit type adenocarcinoma, and mucous columnar cell change is a precursor lesion of pulmonary adenocarcinoma. Modern Pathology (2012) 25, 1265-1274; doi:10.1038/modpathol.2012.76; published online 4 May 2012
\end{abstract}

Keywords: adenocarcinoma; lung; mucous columnar cell change

Most carcinomas, including squamous cell carcinoma in the head and neck, uterine cervix, and skin, and adenocarcinomas in sites including the gastrointestinal tract, biliary tract, breast, and prostate occur through a sequence of steps from normal epithelium to hyperplasia, dysplasia, carcinoma in situ, and invasive carcinoma. ${ }^{1}$ In the lung, squamous carcinoma arises by a similar pathway, beginning from squamous metaplasia in bronchus or bronchiole that is caused by various irritants. ${ }^{2}$ Adenocarcinoma is considered to develop by a pathway of atypical adenomatous hyperplasia and

Correspondence: Dr DH Shin, MD, Department of Pathology, School of Medicine, Pusan National University, Beomeo-ri, Mulgeum-eup, Yangsan 626-770, Korea.

E-mail: donghshin@chol.com

${ }^{4}$ These are co-first authors.

Received 25 January 2012; revised 6 March 2012; accepted 6 March 2012; published online 4 May 2012 bronchioloalveolar carcinoma. ${ }^{2-4}$ However, whether atypical adenomatous hyperplasia-bronchioloalveolar carcinoma pathway is really the only pathway for pulmonary adenocarcinoma has been questioned on the basis of a number of observations. ${ }^{5}$ First, atypical adenomatous hyperplasias are not as common as adenocarcinomas with features of bronchioloalveolar carcinoma or premalignant lesions in other organs. ${ }^{3,5}$ Second, atypical adenomatous hyperplasia is morphologically different from pre-adenocarcinoma lesions in other organs such as adenoma, prostatic intraepithelial neoplasia, or biliary intraepithelial neoplasia showing nuclear stratification, elongation, and overlap. Third, peripheral pulmonary parenchyma in which atypical adenomatous hyperplasia or bronchioloalveolar carcinoma is typically present is the furthest for carcinogens to reach. On the other hand, bronchial or bronchiolar columnar cells are more readily accessible by various carcinogens. Moreover, 
their epithelia are columnar cells as in the organs where adenocarcinoma occurs through premalignant intraepithelial neoplasia, whereas peripheral lung parenchyma consists of flat or cuboidal alveolar cells and atypical adenomatous hyperplasia does not show nuclear stratification, overlap, or elongation. Only a few studies have focused on this issue. ${ }^{5-10}$ Recently, peripheral adenocarcinoma has been defined as terminal respiratory unit adenocarcinoma. ${ }^{11-13}$ Their clinicopathological characteristics are generally well defined. ${ }^{11-13}$ On the contrary, non-terminal respiratory unit type adenocarcinomas have not been well described.

Mucous columnar cells have been suspected as being a precursor lesion of mucinous bronchioloalveolar carcinoma, especially when arising in congenital cystic adenomatoid malformation. ${ }^{14-17}$ Mucous or goblet cell proliferation has been observed in or around congenital cystic adenomatoid malformation, and those mucous columnar cells have genetic abnormalities such as loss of heterozygosity at $\mathrm{P} 16^{\mathrm{INK} 4}$ or gain/loss of chromosomes, in contrast to relatively normal lungs. ${ }^{15}$ On the basis of our recent experience of an adenocarcinoma that displayed an extensive transition from normal ciliated columnar cell to mucous columnar cells, indicative of a spectrum from dysplastic to malignant morphology, we were compelled to consider the possibility that mucous columnar cell metaplasia and dysplasia is another obvious pathway of pulmonary adenocarcinoma. Because mucous columnar cell metaplasia arises in the bronchus or bronchiole, it seems reasonable to think that adenocarcinoma arising through mucous columnar cell change belongs to the so-called central adenocarcinoma or non-terminal respiratory unit type adenocarcinoma, and not to the terminal respiratory unit type adenocarcinoma or peripheral adenocarcinoma.

To explore this further, we reviewed and analyzed our archive files of lung adenocarcinoma displaying mucous columnar cell metaplasia and dysplasia.

\section{Materials and methods}

\section{Case Selection and Slide Review}

Among the series of consecutive non-small cell cancer cases that had been obtained through wedge resection, lobectomy, or pneumonectomy from 2005 to 2010 in Pusan National University Hospital, we selected definite adenocarcinomas that could be diagnosed as adenocarcinoma by histology alone on hematoxylin-eosin-stained slides. Because poorly differentiated adenocarcinomas are usually diagnosed as adenocarcinomas depending on immunohistochemistry, TTF1-negative poorly differentiated adenocarcinomas can be excluded from the study. Hence, we did not include them in this study. A total of 157 cases were chosen. The obtained slides were all separately reviewed by three pathol- ogists (WYP, DHS, and CHL). First, we divided the adenocarcinomas into two groups, terminal respiratory unit and non-terminal respiratory unit, according to the criteria explained in a previous report. ${ }^{10-13}$ Briefly, Clara cells, type II pneumocytes, or bronchiolar cells were subcategorized as terminal respiratory unit type, based on their dome-shaped protruding cytoplasm. Bronchial surface epithelium, mucous cell, or goblet cell morphology was included in the non-terminal respiratory unit type; these cells have flat cytoplasmic surface or mucuscontaining cytoplasm. Second, we investigated the presence of mucous columnar cell change that is transited from normal ciliated columnar cell. $\mathrm{Mu}-$ cous columnar cell was defined as cells having mucin or bluish hue in cytoplasm and losing their cilia.

Pathology reports and clinical records of all the enrolled cases were reviewed. Sex, age, smoking history, and status of epidermal growth factor receptor $(E G F R)$ and KRAS mutations were taken.

\section{Immunohistochemistry}

All specimens used were $3 \mu$ m-thick sections of paraffin-embedded tissue obtained at surgery. Sections were transferred to poly-L-lysine-coated glass slides and air-dried overnight at $37^{\circ} \mathrm{C}$. They were then dewaxed in xylene (three changes), rehydrated in a graded series of decreasing ethanol concentrations, and rinsed in $\mathrm{pH}$ 7.4 Tris-buffered saline. Endogenous peroxidase activity was inactivated with 5\% hydrogen peroxide in methanol for $15 \mathrm{~min}$ at room temperature. Antigen retrieval was performed using $5 \mathrm{~min}$ microwave treatment in TBS. TTF1 (8G7G3, DAKO, Copenhagen, Denmark), CK7 (OV-TL 12/30, DAKO), CK20 (K 20.8, DAKO), CDX2 (DAK-CDX2, DAKO), and ALK1 (ALK1, DAK) were used as the primary antibody. It was incubated for $1 \mathrm{~h}$ at room temperature. The Envision detection kit (DakoCytomation) was used as the secondary antibody. Reaction products were visualized by exposing sections to diaminobenzidine. Nuclei were lightly counterstained for $\sim 20 \mathrm{~s}$ with Mayer's hematoxylin. Only when TTF1, CK7, CK20, CDX2, or ALK1 staining was over $10 \%$ of tumor cells with moderate or strong intensity was TTF1 considered as positive.

\section{Survival Analysis}

Patient survival was calculated as the time between operation and death. Patients who were still alive at the time of data collection were censored in the statistical analyses. The cases lost to follow-up or deaths from any other cause were defined as censored data for the analysis of survival rates. The survival curves were plotted using the KaplanMeier method, and $P$-values were calculated using the log-rank test. 


\section{Results}

Terminal Respiratory Unit versus Non-Terminal Respiratory Unit Type Adenocarcinoma

Among the 157 cases of adenocarcinoma, 121 cases showed the terminal respiratory unit type adenocarcinoma morphology. They showed a mixed growth pattern, including acinar (90\%), lepidic growth $(83 \%)$, micropapillary $(31 \%)$, papillary $(25 \%)$, and solid $(14 \%)$, in descending order of frequency. The cellular morphology of terminal respiratory unit type adenocarcinoma was Clara cell or type II pneumocyte. Their cytoplasm was dome shaped or had an apical protrusion. Mucous columnar cells were absent in terminal respiratory unit type adenocarcinoma. Mucous columnar cell changes are not observed in tumor or adjacent normal lung. TTF1 staining was almost always positive for terminal respiratory unit type adenocarcinoma and was diffuse and intense in the nuclei. Non-terminal respiratory unit type adenocarcinoma comprised 36 cases including 6 pure mucinous bronchioloalveolar carcinomas and 1 colloid adenocarcinoma. Other cases usually showed an acinar type adenocarcinoma mixed with a micropapillary, papillary, solid, or bronchioloalveolar carcinoma component, whereas their tumor cell morphology was goblet cell, mucous columnar cell, or bronchial surface cell. TTF1 staining was never over $50 \%$ in proportion and staining intensity was always weak. In the terminal respiratory unit type adenocarcinoma group, EGFR and KRAS mutations were detected in 56 and 9 cases (46 and $7 \%)$, respectively, whereas $3(8 \%)$ and $9(24 \%)$ cases, respectively, were present in the non-terminal respiratory unit type adenocarcinoma. The clinicopathological information is summarized in Table 1.

\section{Mucous Columnar Cell Change}

In all, 24 cases of non-terminal respiratory unit type adenocarcinoma revealed transition foci from normal ciliated columnar epithelium to mucous columnar cell, showing progression of nuclear atypia to a degree sufficient to permit designation as adenocarcinoma in situ. Normal bronchial epithelium displayed many ciliated columnar cells, and pseudostratification and round to oval nuclei. On transition to mucous columnar cells, their cytoplasm contained mucin vacuoles or became bluish and they lost their cilia. Nuclei were basally located. When they start to transition to dysplastic mucous columnar cells, their nuclei maintained their basal orientation with stratification and were mildly enlarged with fine chromatin and inconspicuous nucleoli (Figure 1). These subtle changes may be regarded as low-grade dysplasia. On progression of dysplasia, mucous columnar cells contained more enlarged nuclei with irregular nuclear membrane, conspicuous nucleoli, and occasional
Table 1 Summary of the cases

\begin{tabular}{|c|c|c|c|}
\hline & $\begin{array}{l}\text { TRU } \\
\text { type }\end{array}$ & $\begin{array}{l}\text { Non-TRU } \\
\text { type }\end{array}$ & P-value \\
\hline Number of cases & 121 & 36 & \\
\hline Median age & 62 & 61 & 0.51 \\
\hline $\operatorname{Sex}(\mathrm{M} / \mathrm{F})$ & $53 / 68$ & $20 / 16$ & 0.17 \\
\hline \multicolumn{4}{|l|}{ T stage } \\
\hline 1 & 75 & 13 & 0.01 \\
\hline 2 & 42 & 19 & \\
\hline 3 & 4 & 3 & \\
\hline 4 & 0 & 1 & \\
\hline \multicolumn{4}{|l|}{$N$ stage } \\
\hline 0 & 92 & 27 & 0.29 \\
\hline 1 & 15 & 2 & \\
\hline 2 & 14 & 7 & \\
\hline \multicolumn{4}{|l|}{ Stage } \\
\hline I & 83 & 21 & 0.43 \\
\hline II & 21 & 7 & \\
\hline III & 17 & 8 & \\
\hline TTF1 & $115(95 \%)$ & $4(11 \%)$ & $<0.01$ \\
\hline EGFR mutation & $56(46 \%)$ & $3(9 \%)$ & $<0.01$ \\
\hline KRAS mutation & $9(7 \%)$ & $9(25 \%)$ & $<0.01$ \\
\hline Smoker ${ }^{\mathrm{a}}$ & $39 / 108$ & $16 / 33$ & 0.22 \\
\hline Smoking quantity (PY) & 12 & 25 & 0.04 \\
\hline
\end{tabular}

TRU, terminal respiratory unit; PY, pack years.

${ }^{\mathrm{a}}$ Smoking history was available in 108 and 33 patients from each group.

multinucleation. Nuclear arrangements were disorganized, with some nuclei reaching the apex of the cytoplasm. Cytoplasm depleted mucin, becoming eosinophilic. Architecturally, micropapillary or papillary growth, tufting, and bridging were frequently observed. We designated this change as high-grade dysplasia (Figure 2). Although adenocarcinoma in situ was almost similar and hard to discern from high-grade dysplasia, nuclei seemed to be more vesicular and had condensed chromatin and prominent nucleoli (Figure 2). Microinvasion from adenocarcinoma in situ was observed (Figure 2). Cystic spaces of honeycombing change also showed bronchiolar metaplasia that exhibits mucous columnar cell change. The mucous columnar cell change and their progression toward dysplasia seem to be similar to the scheme of pancreatic intraepithelial neoplasia, which also shows mucinous change. The proposed criterion for mucous columnar cell change grading is summarized in Table 2 and schematic illustrations in Figure 3.

\section{Pathologic and Clinical Features of Adenocarcinoma Arising from Mucous Columnar Cell Change}

Invasive adenocarcinoma was mostly the well to moderately differentiated acinar type (Figure 4). Compared with terminal respiratory unit type adenocarcinoma, their acini were frequently larger. Papillary or micropapillary growth pattern was 

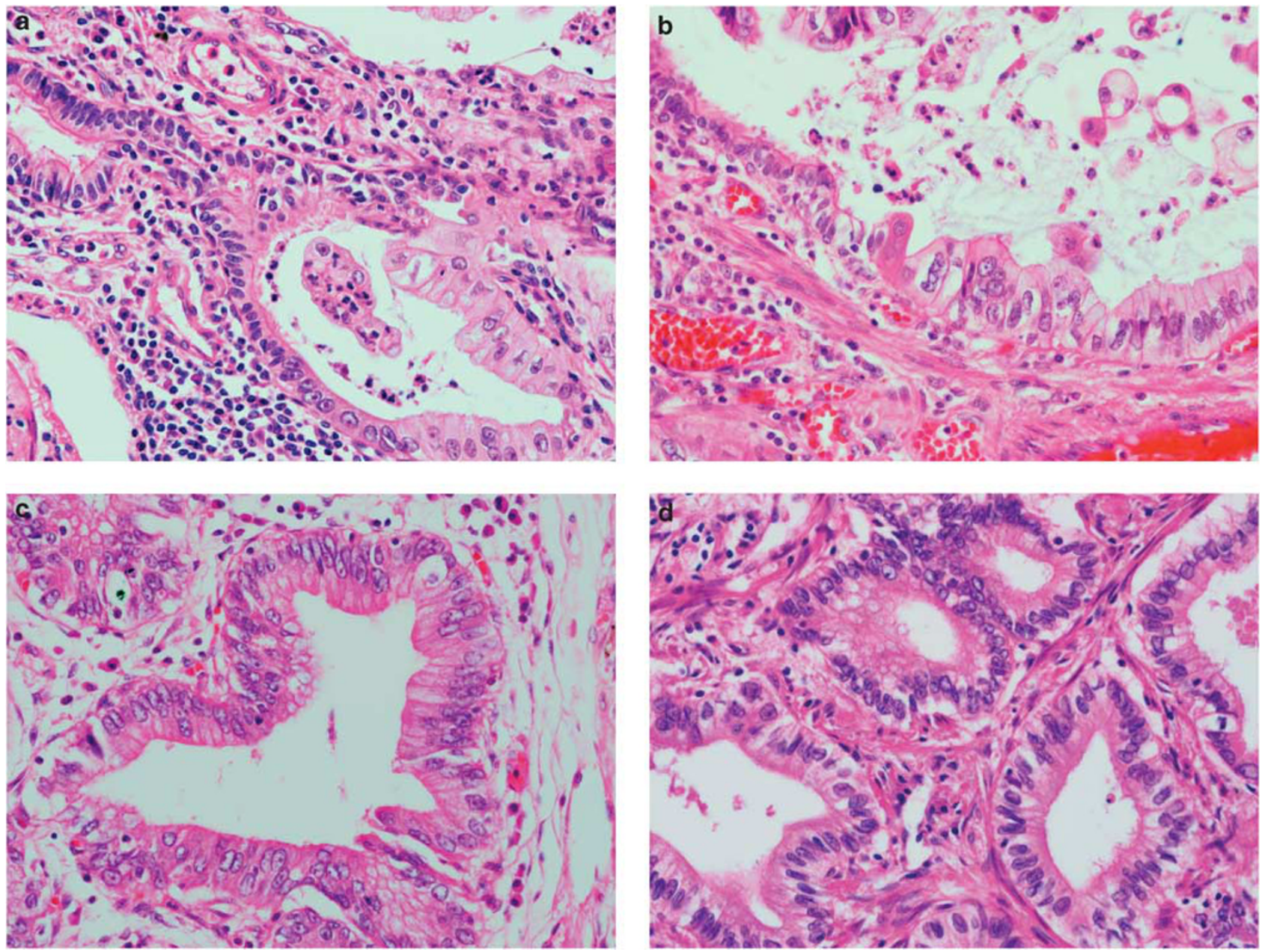

Figure 1 Transition of normal bronchial epithelial cells to mucous columnar cells and low-grade dysplasia. (a, b) Mucous columnar cells have lost their cilia. Mucin of the cytoplasm has increased, appearing bluish. (c, d) Low-grade dysplasia of mucous columnar cells are identified by enlargement, elongation, or overlapping of nuclei. Smooth muscle fibers (d) indicate this area is bronchiole.

occasionally admixed with the acinar type. The lepidic growth pattern, which is the most common pattern in terminal respiratory unit type adenocarcinoma, was not evident. Typical mucinous bronchioloalveolar carcinoma was present in four cases in the main tumor and in three cases in adjacent lung, but they were never the predominant component. Poorly differentiated solid adenocarcinoma coexisted in three cases, squamous cell carcinoma in one case, and pleomorphic carcinoma in one case. Tumor cells of adenocarcinoma arising through mucous columnar cell change consisted of three types, mucous cells, oncocytic cells, and goblet cells, in descending order of frequency. Mucous or oncocytic cells were frequently the predominant cell type, but goblet cells were only focally seen and were never predominant. Especially, oncocytic tumor cells had pleomorphic nuclei with irregular nuclear membrane, vesicular open chromatin, and prominent nucleoli (Figure 4). Some cases showed extensive acute inflammation, necrotic debris, inflammatory cells, and exudates in neoplastic acini or nonneoplastic airway or alveoli (Figure 5). Lymphoid aggregates were also scattered in and outside of the tumors. In all, 10 cases revealed mild to marked honeycombing change in the background of the tumor (Figure 5) and 3 cases also had honeycomb lesions in the adjacent lung parenchyma. The honeycomb spaces were lined by ciliated columnar cells or flattened type II pneumocytes. Transition from normal ciliated columnar cells to dysplastic mucous columnar cells was frequently seen in the honeycomb spaces.

On immunostaining, 24 cases of adenocarcinoma arising from mucous columnar cell change were negative for TTF1 (Figure 6). In normal bronchial mucosa, TTF1 expression was detected in basal cell layers and only focally observed in ciliated columnar cells. When mucous columnar cells appeared and become dysplastic, the basal cell layer disappeared and TTF1 expression was lost or was focally evident (Figure 6). CK7 was consistently positive in all cases. CK20 and CDX2 were positive in four cases, respectively, but were never diffuse (Figure 6). 



Figure 2 Morphology of mucous columnar cells with high-grade dysplasia or carcinomatous change. (a) Mucous columnar cells show piling up and hyperchromatic nuclei. (b) Architectural complexity, papillae and bridging, is seen. (c) Adenocarcinoma in situ has more enlarged and rounder nuclei with occasional prominent nucleoli. Necrotic debris is present. (d) Adenocarcinoma in situ is located in the centrilobular region that is identified by bronchial artery. (e) Microinvasion of adenocarcinoma is observed.

Table 2 Grading of mucous columnar cell change

Group Pathologic description

Normal

Normal bronchial epithelium is pseudostratified and columnar, with numerous ciliated cells and scattered basophilic and flocculent-appearing goblet cells. Basal cells are short cells with relatively little cytoplasm being located along the basement membrane.

Mucous cell metaplasia

These are flat epithelial lesions composed of tall columnar cells with no cilia, basally located nuclei and supranuclear mucin. The nuclei are small and round to oval in shape without dysplasia. Nuclear organization is maintained. The basal cells are absent.

Mucous cell change, low-grade Architecturally, these mucinous epithelial lesions may be flat or rarely papillary. Cytologically, these dysplasia lesions have some nuclear abnormalities. These abnormalities may include some loss of polarity, nuclear crowding, enlarged nuclei, pseudostratification, and hyperchromatism. These nuclear abnormalities fall short of those seen in high-grade dysplasia.

Mucous cell change, high-grade Architecturally, these lesions are usually flat but occasionally papillary or micropapillary. True dysplasia cirbriforming, the appearance of 'budding off' of small clusters of epithelial cells into the lumen, and luminal necrosis are present. Cytologically, these lesions are characterized by a loss of nuclear polarity, nuclear irregularities, and prominent (macro) nucleoli.

Mucous cell change, adenocarcinoma in situ

The lesions resemble carcinoma at the cytonuclear level, but invasion through the basement membrane is absent.

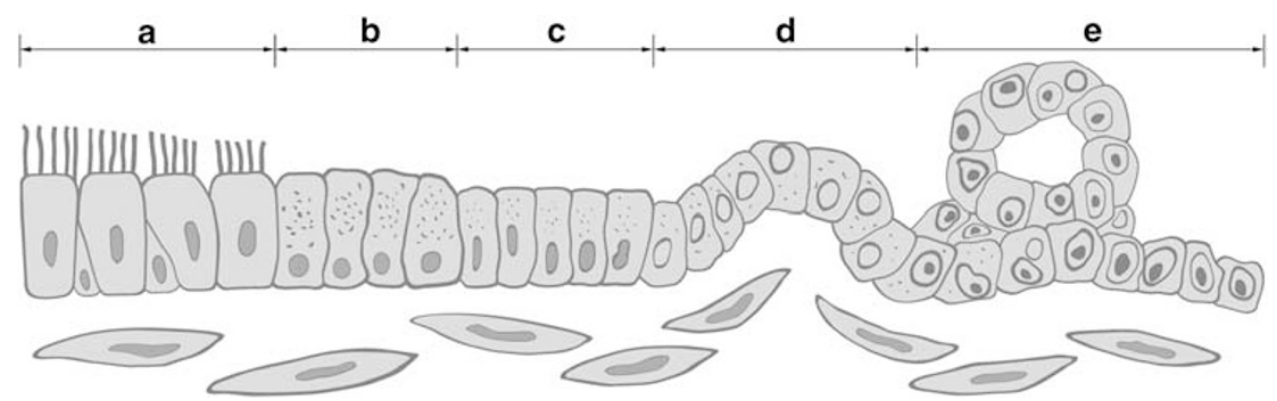

Figure 3 Schematic illustration of mucous columnar cell change. (a) Normal ciliated bronchial cells. (b) Mucous columnar cell metaplasia. (c) Mucous columnar cell change showing low-grade dysplasia. (d) Mucous columnar cell change showing high-grade dysplasia. (e) Adenocarcinoma in situ arising from mucous columnar cell change. 

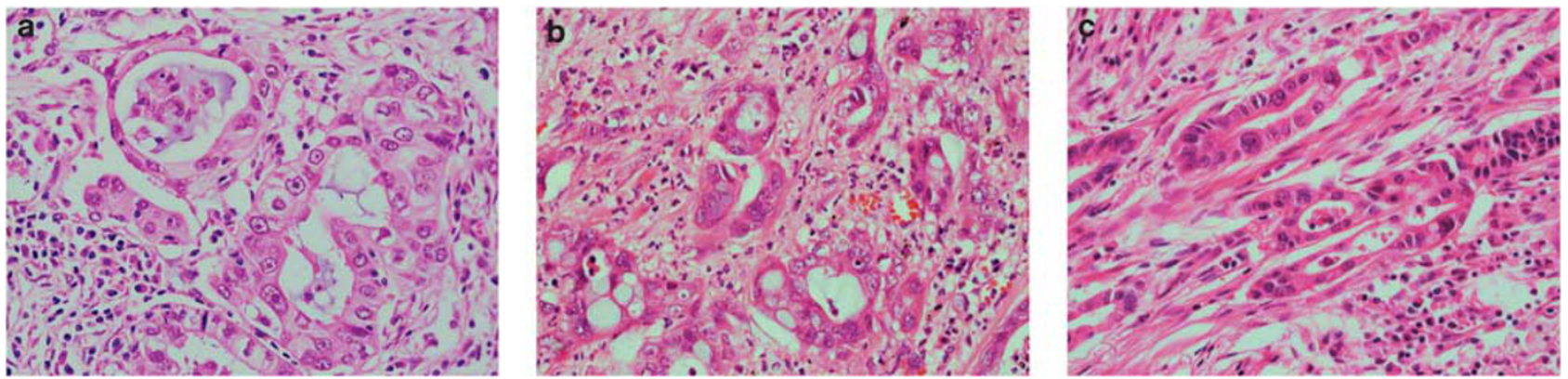

Figure 4 Morphology of combined adenocarcinoma. (a) Adenocarcinoma shows vesicular nuclei with prominent nucleoli. Cytoplasm is pretty eosinophilic. (b) Sometimes, intracellular mucin or mucin inspissate is evident. (c) Adenocarcinoma is architecturally acinar type in most of the cases.
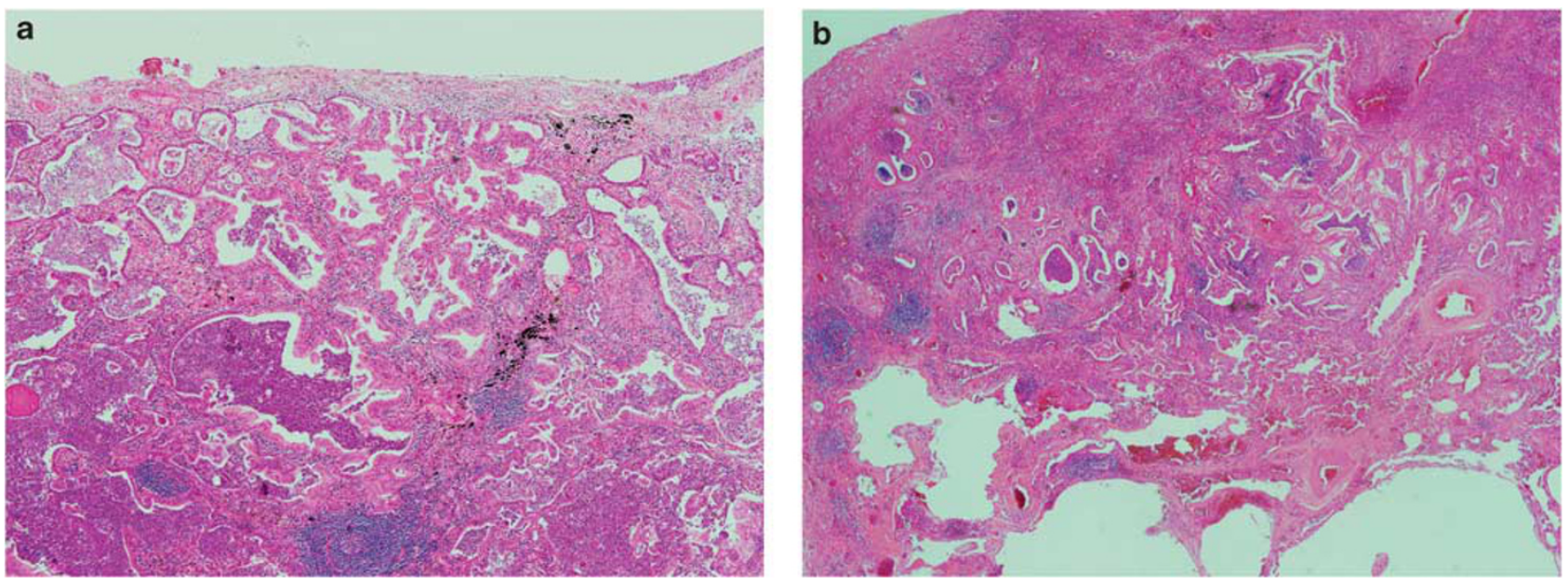

Figure 5 Other combined features. (a) Some cases showed extensive acute inflammation, necrotic debris, inflammatory cells, and exudates, which are present in neoplastic acini or nonneoplastic airway or alveoli. (b) Nine cases revealed mild to marked honeycombing change in the background of tumor.

ALK1 was detected in one case (Figure 6). These 24 cases comprised 14 male and 10 female patients. Twelve patients were smokers, averaging 30 pack years in quantity. EGFR and KRAS mutations were detected in three and six cases, respectively. The clinicopathological findings of these 24 cases are summarized in Table 3.

\section{Survival Analysis}

The mean follow-up duration of 157 patients was 28 months (28.22 \pm 15.99$)$. Twenty patients were dead. Among them, 14 were the patients who had terminal respiratory unit type adenocarcinoma and 6 were those who had non-terminal respiratory unit type adenocarcinoma. Survival difference between terminal respiratory unit type and non-terminal respiratory unit type patients was not significant statistically (Figure 7).

\section{Discussion}

Historically, pulmonary adenocarcinoma arising in sporadic cases of congenital cystic adenomatoid malformation has been the main clinicopathological setting in which mucous columnar cell change has been reported. ${ }^{14-17}$ In the current study, we found 24 cases of adenocarcinoma displaying transition foci from the normal ciliated columnar cell to mucous columnar cell metaplasia, dysplasia, adenocarcinoma in situ, and finally invasive adenocarcinoma. Transition foci of normal ciliated columnar epithelium to mucous columnar cell were observed in bronchiole or honeycomb spaces. Normal ciliated columnar cells lost their cilia and showed disorientation and enlarged vesicular nuclei with occasional multinucleation. The cellular morphology of dysplastic mucous columnar cell and invasive adenocarcinoma was very similar. Both lesions displayed cytoplasm containing mucin with pale to light pinkish color similar to gastric foveolar epithelial cells. Shimosato ${ }^{10}$ classified pulmonary adenocarcinomas cytologically into five cell types: cells resembling bronchial goblet cells, bronchial gland cells, bronchial surface epithelial cells, type II pneumocytes, and nonciliated bronchiolar epithelial cells. More recently, Inamura ${ }^{18}$ simplified this scheme into hobnail, columnar, and polygonal cells. Terminal respiratory unit type adenocarcinoma 



Figure 6 Result of immunohistochemistry. Mucous columnar cells lose TTF1 expression (a) compared with normal ciliated bronchial cells. Mucous columnar cells with low-grade dysplasia did not express TTF1 (b) and combined adenocarcinoma is also negative for TTF1 (c), but express CK7 diffusely (d). Some cases showed CK20 (e) or CDX2 (f) expression. One case exhibited ALK1 positivity in mucous columnar cells (g).

develops from bronchiolar epithelial cells, Clara cells, or type II pneumocytes, which is hobnail cell. ${ }^{10,12-14}$ The morphology of adenocarcinoma in this study may be classified as columnar cell type, which was explained as having mucous or goblet cell. ${ }^{18,19}$ Most characteristically, all these adenocarcinomas arising through mucous columnar cell change were negative for TTF1. TTF1 expression in the basal layer of the bronchial epithelium was lost when ciliated columnar epithelium is replaced by mucous columnar cells. Among the 24 cases, 9 had mild to marked honeycombing change in the background and 17 cases were associated with mild to intense acute inflammation, with inflammatory exudates being present in the neoplastic glands. In honeycomb spaces, pneumocytes are lost and bronchial metaplasia covers the honeycomb spaces, followed by mucous columnar cell metaplasia, dysplasia, and, finally, adenocarcinoma. This event may be the same mechanism involved in mucinous bronchioloalveolar carcinomas arising from conge- nital cystic adenomatoid malformation. In this case, the center of adenocarcinoma may reside in peripheral lung parenchyma. Actually, in 7 out of 24 cases, typical mucinous bronchioloalveolar carcinoma coexisted in the adjacent lung. This observation indicates that the same etiology may be involved in the development of adenocarcinoma arising through mucous columnar cell change and mucinous bronchioloalveolar carcinoma. However, mucous columnar cells and their matched adenocarcinoma did not show predominant goblet cell morphology that is frequently evident in mucinous bronchioloalveolar carcinoma, and mucinous bronchioloalveolar carcinomas were only focally existed. This observation is very akin to recent description about columnar cell type adenocarcinoma. ${ }^{18,19}$ The characteristics of terminal respiratory unit type adenocarcinoma are highlighted by morphologic resemblance to type II pneumocytes, Clara cells, and/or nonciliated bronchiolar cells, and feature the constant and 
Table 3 Clinicopathological characteristics of adenocarcinoma with transition foci from normal ciliated columnar cells to mucous columnar cell change



\begin{tabular}{|c|c|c|c|c|c|c|c|c|c|c|c|c|c|c|c|}
\hline 1 & $71 / \mathrm{M}$ & 7.5 & II & $\mathrm{A}, \mathrm{mP}, \mathrm{PD}$ & ++ & +++ & - & + & + & - & - & - & + & 80 & DOD (70) \\
\hline 2 & $60 / F$ & 2.4 & III & A, solid & + & + & - & + & - & - & - & - & - & 0 & DOD (48) \\
\hline 3 & $59 / \mathrm{M}$ & 11 & III & $\mathrm{A}, \mathrm{mBAC}$ & + & + & - & + & - & - & - & - & - & 60 & DOO (2) \\
\hline 4 & $80 / \mathrm{M}$ & 1.5 & III & A, PD & ++ & ++ & - & + & - & - & - & - & + & 50 & AW (24) \\
\hline 5 & $80 / \mathrm{M}$ & 2.8 & III & $\mathrm{A}$ & +++ & - & - & + & - & - & - & - & - & 50 & AW (24) \\
\hline 6 & $54 / F$ & 1.2 & I & $\mathrm{A}, \mathrm{mP}$ & - & - & - & + & - & - & - & - & - & 0 & AW (67) \\
\hline 7 & $66 / \mathrm{M}$ & 4.0 & $\mathrm{I}$ & A, pap & + & - & - & + & - & - & + & - & - & 40 & AW (63) \\
\hline 8 & $81 / \mathrm{M}$ & 5.0 & III & A, solid & +++ & + & - & + & - & - & - & - & - & 100 & DOD (32) \\
\hline 9 & $55 / \mathrm{F}$ & 3.5 & $\mathrm{I}$ & $\mathrm{A}, \mathrm{mBAC}, \mathrm{PL}$ & ++ & - & - & + & - & - & - & - & + & 0 & AW (63) \\
\hline 10 & $70 / \mathrm{F}$ & 3.4 & $\mathrm{I}$ & $\mathrm{A}$ & + & + & - & + & + & - & - & - & - & 0 & AW (47) \\
\hline 11 & $70 / \mathrm{M}$ & 5.0 & $\mathrm{I}$ & A, solid & + & - & - & + & - & - & - & - & + & 0 & AW (32) \\
\hline 12 & $60 / \mathrm{M}$ & 6.0 & II & A, mP, Pap & +++ & + & - & + & + & + & - & - & - & 90 & AW (26) \\
\hline 13 & $60 / \mathrm{F}$ & 6.0 & II & $\mathrm{A}, \mathrm{mP}$ & - & + & - & + & - & - & - & - & - & 0 & DOD (23) \\
\hline 14 & $77 / \mathrm{M}$ & 1.2 & $\mathrm{I}$ & A, LG & - & - & - & + & - & - & - & - & - & 0 & AW (23) \\
\hline 15 & $67 / \mathrm{F}$ & 2.2 & $\mathrm{I}$ & A, SCC & + & + & - & + & - & - & - & + & - & 0 & AW (23) \\
\hline 16 & $77 / \mathrm{F}$ & 3.3 & $\mathrm{I}$ & $\mathrm{A}$ & - & + & - & + & - & - & - & - & - & 0 & AW (7) \\
\hline 17 & $61 / \mathrm{M}$ & 10.0 & III & A, mBAC, Pap & - & + & - & + & - & + & - & + & - & 60 & AWD (19) \\
\hline 18 & $69 / \mathrm{M}$ & 3.9 & $\mathrm{I}$ & $\mathrm{A}, \mathrm{mBAC}$ & ++ & - & - & + & - & - & - & - & + & 45 & AW (21) \\
\hline 19 & $64 / \mathrm{M}$ & 3.2 & $\mathrm{I}$ & $\mathrm{A}$ & + & ++ & - & + & + & + & - & - & - & 90 & AW (16) \\
\hline 20 & $55 / \mathrm{F}$ & 5.5 & II & $\mathrm{A}$ & + & + & - & + & - & + & - & - & + & 0 & AW (14) \\
\hline 21 & $52 / \mathrm{F}$ & 2.4 & $\mathrm{I}$ & $\mathrm{A}$ & - & + & - & + & - & - & - & - & - & 0 & AW (15) \\
\hline 22 & $53 / \mathrm{M}$ & 7.2 & III & A, mP, pap & ++ & + & - & + & - & - & - & - & - & 30 & AWD (12) \\
\hline 23 & $56 / \mathrm{F}$ & 4.5 & $\mathrm{I}$ & $\mathrm{A}$ & - & +++ & - & + & - & - & - & + & - & 0 & AW (12) \\
\hline 24 & $60 / \mathrm{M}$ & 6.5 & II & $\mathrm{A}$ & +++ & +++ & - & + & - & - & - & - & - & 30 & AW (7) \\
\hline
\end{tabular}

HC, honeycomb change; Inflam, inflammation; EGFR, epidermal growth factor receptor; A, acinar; mP, micropapillary; mBAC, mucinous bronchioloalveolar carcinoma; PD, poorly differentiate; pap, papillary; PL, pleomorphic carcinoma; LG, lepidic growth; SCC, squamous cell carcinoma; PY, pack years; +, mild; ++, moderate; +++, marked; -, absent; AW, alive and well; AWD, alive with disease; DOO, died of other cause; DOD, died of disease.

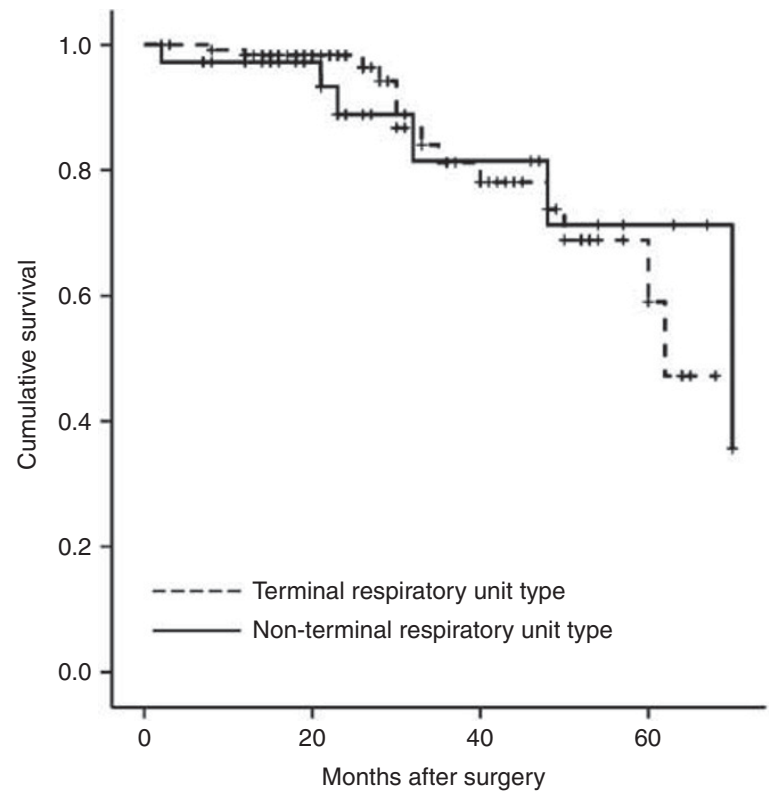

Figure 7 Overall survival curves of terminal respiratory unit and non-terminal respiratory unit adenocarcinomas. Terminal respiratory unit and non-terminal respiratory unit adenocarcinomas did not show survival difference.

uniform expression of TTF1. Nonmucinous bronchioloalveolar carcinoma, mixed adenocarcinomas with bronchioloalveolar carcinoma component, and a subset of papillary carcinoma belong to the terminal respiratory unit type. They show frequent EGFR mutation and no KRAS mutation. Clinically, they are presented in non-smoker Asian females. Adenocarcinoma described in the present study as arising from mucous columnar cell change can be defined as non-terminal respiratory unit type adenocarcinoma based on the following observations. First, its morphology is not included in terminal respiratory unit type adenocarcinoma, showing columnar cell morphology. Second, it is negative for TTF1. TTF1 is the most sensitive and specific marker to define terminal respiratory unit type adenocarcinoma. ${ }^{11}$ Third, EGFR mutation was observed in $8 \%$ of the cases. In one study, EGFR mutation rate of terminal respiratory unit type adenocarcinoma was $61 \%$ and that of non-terminal respiratory unit type was $13 \%$. Some mentions of non-terminal respiratory unit type adenocarcinoma or central bronchial adenocarcinoma have been made. ${ }^{7-10}$ An old study by Edwards ${ }^{8}$ suggested three subtypes of adenocarcinoma according to its location: groups I to III. Group I was designated as parenchymal adenocarcinoma and group II as bronchial adenocarcinoma. Among group II, the majority was 'bronchial acinar adenocarcinoma', cells of which are described as mucus cells having granular, deeply eosinophilic or amphophilic cytoplasm. One recent article suggested three 
possible origin of non-small cell lung cancer: airway progenitor cells (basal cells), bipotential progenitor cells (Clara cells), and alveolar progenitor cells (type II pneumocytes). ${ }^{20}$ Terminal respiratory unit type adenocarcinoma would match with the second or third and non-terminal respiratory unit type with the first. Mucous columnar cell lesions could be regarded as the first type, but better explained by bronchial surface epithelium origin. The recently proposed bronchiolar columnar cell dysplasia is also one of the volunteer precursor lesions of adenocarcinoma. ${ }^{6}$ Bronchiolar columnar cell dysplasia is seen in peribronchiolar area where ciliated bronchial cells reside. Hence, although bronchiolar columnar cell dysplasia was suggested as a precursor lesion of peripheral adenocarcinoma, bronchiolar columnar cell dysplasia seems to arise from ciliated bronchial type columnar cells. A similar finding of peribronchiolar metaplasia has also been observed in peribronchial area adjacent to adenocarcinoma. ${ }^{21}$ The peribronchial columnar cells showed variable degree of atypia ranged from mild to marked.

Our 24 cases of adenocarcinoma arising from mucous columnar cell change had occurred more in males, with 14 males and 10 females. Among the 24 people, 12 were smokers or ex-smokers, averaging 30 pack years in smoking amount. EGFR and KRAS mutations were detected in three and six patients, respectively. Because bronchi or bronchiole are more proximal to lung parenchyma in which type adenocarcinoma arises, direct exposure to tobacco smoke, pollutants, or air-borne chemicals will affect this region first. Mucous columnar cell change may arise in response to these irritants. This hypothesis is also reported by another group who suggested columnar cell adenocarcinoma is related to exposure to exogenic carcinogen. ${ }^{18}$ The reason why mucous columnar cell metaplasia and dysplasia are not seen frequently in lung parenchyma adjacent to adenocarcinoma may be explained by the fact that lung parenchyma occupies a much greater area than the airway. Hence, adenocarcinoma arising through atypical adenomatous hyperplasia and bronchioloalveolar carcinoma may be more frequent. Furthermore, when the airway is exposed to an irritant, squamous metaplasia seems to be the more favored pathway than mucous columnar cell change, being a precursor lesion of squamous cell carcinoma. Adenocarcinoma arising through mucous columnar cell change may have following characteristics more frequently than other adenocarcinoma: (1) presence of transition foci from normal ciliated columnar cells to mucous columnar cell metaplasia and dysplasia in tumor, (2) mucus containing or eosinophilic cytoplasm, (3) TTF1 negativity, and (4) frequent smoker.

In conclusion, we suggest that a group of adenocarcinoma arises through mucous columnar cell change, progressing from mucous columnar cell metaplasia to dysplasia. This group of adenocarci- noma belongs to non-terminal respiratory unit type adenocarcinoma and may have different clinical, histological, immunohistochemical, and molecular traits from the terminal respiratory unit type adenocarcinoma or even other non-terminal respiratory unit type adenocarcinomas. Hence, future investigations are necessary to examine the immunohistochemical or molecular characteristics of this type of adenocarcinoma. Furthermore, this kind of adenocarcinoma can show different responses against various therapeutic modalities.

\section{Acknowledgements}

This research was supported by Basic Science Research Program through the National Research Foundation of Korea (NRF) funded by the Ministry of Education, Science and Technology (2011-0012351).

\section{Disclosure/conflict of interest}

The authors declare no conflict of interest.

\section{References}

1 Stricker TP, Kumar V. Neoplasia. In: Kumar V, Cotran RS, Robbins SL (eds). Robbins Basic Pathology. Saunders: Philadelphia, PA, 2010, pp 325-360.

2 Kohno H, Hiroshima K, Toyozaki T, et al p53 mutation and allelic loss of chromosome 3p, 9p of preneoplastic lesions in patients with nonsmall cell lung carcinoma. Cancer 1999;85:341-347.

3 Mori M, Rao SK, Popper HH, et al Atypical adenomatous hyperplasia of the lung: a probable forerunner in the development of adenocarcinoma of the lung. Mod Pathol 2001;14:72-84.

4 Chapman A, Kerr K. The association between atypical adenomatous hyperplasia and primary lung cancer. Br J Cancer 2000;83:632-636.

5 Mori M, Rao SK, Popper HH, et al Atypical adenomatous hyperplasia of the lung: a probable forerunner in the development of adenocarcinoma of the lung. Mod Pathol 2001;14:72-84.

6 Ullmann R, Bongiovanni M, Halbwedl I, et al Bronchiolar columnar cell dysplasia-genetic analysis of a novel preneoplastic lesion of peripheral lung. Virchows Arch 2003;442:429-446.

7 Pankiewicz W, Minarowski L, liñska W, et al Immunohistochemical markers of cancerogenesis in the lung. Folia Histochem Cytobiol 2007;45:65-74.

8 Edwards CW. Pulmonary adenocarcinoma: review of 106 cases and proposed new classification. J Clin Pathol 1987;40:125-135.

9 Stenhouse G, Fyfe N, King G, et al Thyroid transcription factor 1 in pulmonary adenocarcinoma. J Clin Pathol 2004;57:383-387.

10 Shimosato Y. Bronchial gland type carcinomas. In Corrin B (ed). Pathology of Lung Tumours. Churchill Livingstone: Edinburgh, 1997, pp 135-147. 
11 Yatabe Y, Mitsudomi T. Epidermal growth factor receptor mutations in lung cancers. Pathol Int 2007; 57:233-244.

12 Yatabe Y, Kosaka T, Takahashi T, et al EGFR mutation is specific for terminal respiratory unit type adenocarcinoma. Am J Surg Pathol 2005;29:633-639.

13 Yatabe Y, Mitsudomi T, Takahashi T. TTF-1 expression in pulmonary adenocarcinomas. Am J Surg Pathol 2002;26:767-773.

14 Ramos SG, Barbosa GH, Tavora FR, et al Bronchioloalveolar carcinoma arising in a congenital pulmonary airway malformation in a child: case report with an update of this association. J Pediatr Surg 2007; 42:E1-E4.

15 Stacher E, Ullmann R, Halbwedl I, et al Atypical goblet cell hyperplasia in congenital cystic adenomatoid malformation as a possible preneoplasia for pulmonary adenocarcinoma in childhood: a genetic analysis. Hum Pathol 2004;35:565-570.

16 Yoo JY, Lee SM, Jung JH, et al Adenocarcinoma arising in type 1 congenital cystic adenomatoid malformation: a case report and review of the literature. Kor J Pathol 2008;42:396-400.

17 Lantuejoul S, Nicholson AG, Sartori G, et al Mucinous cells in type 1 pulmonary congenital cystic adenomatoid malformation as mucinous bronchioloalveolar carcinoma precursors. Am J Surg Pathol 2007;31:961-969.

18 Inamura $\mathrm{K}$, Ninomiya $\mathrm{H}$, Ishikawa $\mathrm{Y}$, et al Is the epidermal growth factor receptor status in lung cancers reflected in clinicopathologic features? Arch Pathol Lab Med 2010;134:66-72.

19 Kerr KM. Pulmonary adenocarcinomas: classification and reporting. Histopathology 2009;54:12-27.

20 Chilosi M, Murer B. Mixed adenocarcinomas of the lung: place in new proposals in classification, mandatory for target therapy. Arch Pathol Lab Med 2010;134:55-65.

21 Jensen-Taubman SM, Steinberg SM, Linnoila RI. Bronchiolization of the alveoli in lung cancer: pathology, patterns of differentiation and oncogene expression. Int J Cancer 1998;75:489-496. 